\title{
Hepatitis $C$ virus infection, cryoglobulinemia, and peripheral neuropathy: a case report
}

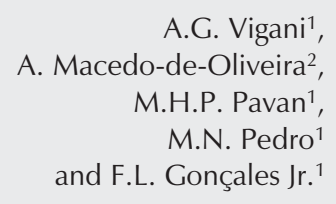

A.G. Vigani ${ }^{1}$,

A. Macedo-de-Oliveira²,

M.H.P. Pavan ${ }^{1}$,

M.N. Pedro ${ }^{1}$

and F.L. Gonçales Jr. ${ }^{1}$

\author{
${ }^{1}$ Grupo de Estudo das Hepatites Virais, ${ }^{2}$ Disciplina de Infectologia, \\ Departamento de Clínica Médica, Faculdade de Ciências Médicas, \\ Universidade Estadual de Campinas, Campinas, SP, Brasil
}

\section{Correspondence \\ A.G. Vigani \\ Departamento de Clínica Médica \\ Hospital de Clinicas, UNICAMP \\ Caixa Postal 6198 \\ 13083-970 Campinas, SP \\ Brasil \\ Fax: +55-19-3788-7727 \\ E-mail: viganialine@hotmail.com}

Publication supported by FAPESP.

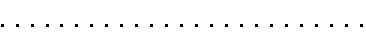

Received February 23, 2005

Accepted August 12, 2005

\begin{abstract}
Hepatitis $\mathrm{C}$ virus (HCV) is essentially hepatotropic but its manifestations can extend beyond the liver. It can be associated with autoimmune diseases, such as mixed cryoglobulinemia, membranoproliferative glomerulonephritis, autoimmune thyroiditis, and lymphoproliferative disorders. The mechanisms that trigger these manifestations are not completely understood. We describe a 48-year-old man with chronic HCV infection (circulating HCV RNA and moderate hepatitis as indicated by liver biopsy), cryoglobulinemia, and sensory and motor peripheral neuropathy. The diagnosis of multineuropathy was confirmed by clinical examination and electromyographic tests. A nerve biopsy revealed an inflammatory infiltrate in the perineurial space and signs of demyelination and axonal degeneration. The patient had no improvement of neurological symptoms with the use of analgesics and neuro-modulators. He was then treated with interferon$\alpha$ (3 million units subcutaneously, 3 times per week) and ribavirin (500 mg orally, twice a day) for 48 weeks. Six months after the end of therapy, the patient had sustained viral response (negative HCV RNA) and remission of neurological symptoms, but cryoglobulins remained positive. A review of the literature on the pathogenesis and treatment of neurological manifestations associated with HCV infection is presented. This report underscores the need for a thorough evaluation of $\mathrm{HCV}$-infected patients because of the possibility of extrahepatic manifestations. Antiviral treatment with interferon and ribavirin can be effective and should be considered in patients with neurological complications associated with HCV infection.
\end{abstract}

\section{Introduction}

Hepatitis $\mathrm{C}$ virus (HCV) infection affects approximately 170 million persons worldwide and is a pandemic 5 times larger than that of HIV. In the United States and Brazil, approximately $2 \%$ of the population is sero-
Key words

- Hepatitis C virus

- Neurological manifestations

- Cryoglobulins

- Interferon

- Ribavirin positive for $\mathrm{HCV}$ (1). The most common mode of $\mathrm{HCV}$ transmission is percutaneous exposure to contaminated blood, including situations associated with intravenous drug use and blood transfusions. HCV infection is a common cause of chronic liver disease, cirrhosis, and hepatocellular carcinoma (1). 
In addition to the liver manifestations, chronic HCV infection may be associated with a series of extrahepatic manifestations, such as mixed cryoglobulinemia, membranoproliferative glomerulonephritis, autoimmune thyroiditis, and lymphoproliferative disorders. These manifestations result from lymphoproliferative and/or autoimmune mechanisms, and occur in 40 to $75 \%$ of patients with chronic HCV infection (2).

The association of $\mathrm{HCV}$ infection with mixed cryoglobulinemia and peripheral neuropathy has been previously reported (3). However, its pathogenesis is not completely understood, nor do formal treatment guidelines exist. Peripheral neuropathy and detectable serum cryoglobulins appear in approximately one third of patients with $\mathrm{HCV}$ infection, but $\mathrm{HCV}$-infected patients with peripheral neuropathy in the absence of serum cryoglobulins have also been described $(3,4)$. In this report, we present a patient with chronic HCV infection, cryoglobulinemia, and peripheral neuropathy.

\section{Case report}

A 48-year-old man was referred to the Disciplina de Neurologia of Hospital das Clínicas, Universidade Estadual de Campinas, due to hypoesthesia on the lateral side of the right foot, which progressed to the lower and upper limbs, accompanied by distal paresthesias of both legs. The patient denied arthralgia and Raynaud's phenomenon. He had been treated with bromazepam, prednisone, paracetamol, codeine, and carbamazepine without symptom relief. His medical history was unremarkable; he specifically denied illicit drug use, alcohol abuse, contact with toxic substances, and diabetes. He had been smoking approximately 40 cigarettes per day for 20 years. Neurological examination revealed motor weakness in the lower right limb (graded as 4 in the general medical conditions grading - maximum 5). Areas of anesthesia were found in both hands and feet. Ankle tendon reflexes were abolished.

Laboratory findings revealed normal platelet, red and white blood cell counts. Serum levels of total bilirubin, glucose, electrolytes, creatine, urea nitrogen, alanine aminotransferase, aspartate aminotransferase, alkaline phosphatase, and $\gamma$-glutamyl transferase were all in the normal range. Thyroid function tests and protein electrophoresis did not reveal any abnormality. Anti-DNA, antinuclear, anti-SSA/RO, anti-SSB, antineutrophil cytoplasmic, anti-Jo1, anti-SCL 70 , and anti-ENA antibodies and lupus erythematosus cells were all normal or negative. C-reactive protein $(3.21 \mathrm{mg} / \mathrm{dL}$; normal $<0.5 \mathrm{mg} / \mathrm{dL}$ ) and rheumatoid factor (219 IU/ $\mathrm{mL}$; normal $<20 \mathrm{IU} / \mathrm{mL}$ ) were increased. Urine and cerebrospinal fluid analyses were normal. Serum cryoglobulins were detected (Hospital das Clínicas in-house method), but not typed or quantified.

Electromyography showed a severe and generalized neuropathic impairment mainly in the lower limbs. The evoked potential component revealed compromised activity, predominantly axonal, but myelinic involvement was also present. In addition, asymmetric nerve conduction was demonstrated in the lower extremities. These findings were suggestive of multineuropathy. A biopsy of the right sural nerve revealed myelinic sheath reduction and inflammatory infiltrate in the perineural space. Signs of axonal degeneration were also present (Figure 1).

Serum anti-HCV antibodies detected by enzyme immunoassay (Abbott AxSYM AntiHCV 3.0, Abbott Laboratories, Wiesbaden, Hessen, Germany) were positive. After this result, the patient was referred to the Grupo de Estudo das Hepatites Virais outpatient clinic at Hospital das Clínicas, Universidade Estadual de Campinas, for HCV infection evaluation and follow-up. Serum HCV RNA was detected by qualitative PCR (Cobas Amplicor HCV Test v2.0, Roche Molecular Diagnostic Systems, Branchburg, NJ, USA) 
and HCV genotype 1 was identified. Regarding hepatitis B virus (HBV) infection, anti-HBc antibodies were positive, but HBsAg, anti-HBs, $\mathrm{HBeAg}$, and anti-HBe were all negative. Serum HBV DNA was undetectable by hybridization. Serologies for HIV, syphilis, and Epstein-Barr virus were negative. A liver biopsy showed moderate chronic hepatitis. Portal fibrosis with septa (score F2 by the Metavir scoring system) and necroinflammation (grade A2 by the Metavir scoring system) were present.

The patient was tentatively treated with amitriptyline, phenytoin, and folic acid. Despite treatment, his condition deteriorated: he became unable to walk and presented dysesthesia in sock distribution, which was more pronounced in the distal right leg. Treatment with interferon- $\alpha$ (IFN- $\alpha, 3$ million units subcutaneously, three times per week) and ribavirin (500 $\mathrm{mg}$ orally, twice a day) was initiated. Three months later, the patient experienced arthralgia in large and small joints. Seven months after the beginning of treatment, the neurological symptoms began to improve and, by the end of treatment (a total of 48 weeks), serum HCV RNA was negative. Six months after the end of treatment, the patient had relief of neurological symptoms and negative HCV RNA, which characterizes sustained viral response, but serum cryoglobulins remained positive.

\section{Discussion}

The HCV incubation period varies from 2 to 26 weeks (median 6 weeks). Acute infection is asymptomatic in 60 to $70 \%$ of the patients and 20 to $30 \%$ of infected persons develop jaundice. Approximately 15 to $30 \%$ of patients exposed to HCV recover spontaneously, whereas the remaining patients develop chronic infection. One third of chronically infected patients present moderate to severe hepatitis within 20 years of infection. Inflammation and hepatic cell necrosis may lead to bridging fibrosis, and cirrhosis appears in 15 to $20 \%$ of patients with chronic infection. Antibody tests can be used to detect HCV exposure but they do not differentiate between resolved and chronic infection, a task accomplished with HCV RNA detection tests, such as PCR (1).

Genetic analysis of HCV revealed the existence of six different genotypes, which differ in their genome by up to $35 \%$. Genotype 1 is the most common variant found in the United States and Brazil, but the least responsive to treatment. $\mathrm{HCV}$ genotype 1 infections should be treated with a combination of IFN or pegylated IFN, and ribavirin for 48 weeks. Since genotypes 2 and 3 are more responsive to therapy, the duration of treatment for these genotypes is 24 weeks (1).

Essential mixed cryoglobulinemia (EMC) is a systemic vasculitis disorder characterized by deposition of serum cryoprecipitable (precipitation at temperatures $<37^{\circ} \mathrm{C}$ ) immune complexes in small- and medium-sized blood vessels. Based on immunoglobulin composition, EMC has been divided into type I (exclusively monoclonal), type II (IgM monoclonal and IgG polyclonal), and type III (exclusively polyclonal). Type I cryoglobulinemia does not show rheumatoid factor activity and is usually associated with lymphoproliferative disorders, whereas types II and III are not necessarily associated with these or other disorders, such as autoimmune or chronic liver diseases and viral

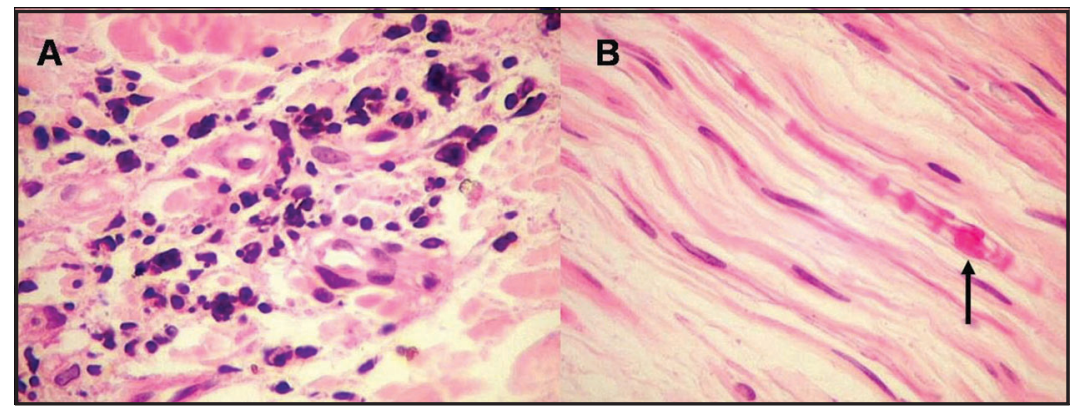

Figure 1. Right sural nerve biopsy stained with hematoxylin and eosin. There is an intense inflammatory infiltrate within the epineurium transverse section (panel A) and reduced myelin sheath-forming Schwann cells in the longitudinal section (panel B). The arrow shows the only preserved axon. $400 \mathrm{X}$. 
infections (e.g., Epstein-Barr virus infections). Types II and III are called mixed cryoglobulinemias and usually show rheumatoid factor activity. Because of high rates of association between EMC and hepatocellular abnormalities, it is speculated that hepatotropic viruses, such as HCV, might be involved in the pathogenesis of the former (5).

The ability of HCV to cause extrahepatic manifestations has been demonstrated previously $(1,6)$. One report has shown serum cryoglobulins in $36-54 \%$ of patients with chronic hepatitis C (7). Lunel et al. (8) found serum cryoglobulins in 72 (57\%) of 127 $\mathrm{HCV}$-infected patients and in only $4 \%$ of uninfected persons. Approximately one third of patients with hepatitis $\mathrm{C}$ and serum cryoglobulins have type II cryoglobulinemia and two thirds have type III. Another study has shown that 81 to $90 \%$ of patients with EMC were HCV RNA positive (9). The mechanism by which HCV elicits cryoglobulin formation is unclear, but may be related to the persistence of $\mathrm{HCV}$ in the cells of the immune system and/or chronic stimulation of the immune response by HCV. Genetic factors, such as human leukocyte antigens, may also be involved in the pathogenesis of HCV-associated cryoglobulinemia.

The majority of patients with cryoglobulinemia are asymptomatic. Only $13-30 \%$ of patients are symptomatic and their clinical presentation varies from mild vasculitis (Raynaud phenomenon (20-50\%), arthralgia (20\%), weakness (65\%), and purpura (10\%)) to severe vasculitis (peripheral neuropathy (15-25\%), membranoproliferative glomerulonephritis (30-50\%), and systemic vasculitis $(8 \%))(9,10)$. The reason for such wide variation of symptoms and organ involvement is not completely understood.

Sensory and/or motor peripheral neuropathy has been found in up to $9 \%$ of patients with chronic HCV infection (6). HCV-associated peripheral neuropathy is mainly related to axonal damage and cryoglobuline- mia is usually present (11). Previous studies have described this peripheral neuropathy as both a motor and sensory polyneuropathy, mainly distal, and of subacute onset; although cases of exclusively asymmetrical sensory neuropathy have also been reported (12-14). The presence of serum cryoglobulins is predictive of more severe and widespread neuropathic involvement $(4,11)$.

In this report, we describe a patient with $\mathrm{HCV}$-associated cryoglobulinemia and peripheral neuropathy whose neurological manifestation was a multineuropathy of insidious progression. A nerve biopsy showed axonal degeneration and inflammatory infiltration in the perineurial space, which is in accordance with previous reports $(11,15)$.

Peripheral neuropathy usually occurs in types II and III, rather than in type I, EMC. Two main pathogenic mechanisms have been suggested: 1) impairment of the vasa nervorum microcirculation by intravascular deposits of cryoglobulins, producing ischemia due to derangement of the microcirculation, and 2) necrotizing vasculitis induced by longstanding cryoglobulin precipitation, complement fixation, and rheumatoid factor activity. Either the vasculitis itself or the vascular occlusion causes fascicular ischemia, which ultimately results in axonal degeneration. Some investigators speculate that the first mechanism may account for the pattern of progressive sensory polyneuropathy and the second for the multineuropathy features (16). Demyelinating neuropathy, such as the one seen in this patient, has been reported previously in few HCV-infected patients. Immune complex deposition on the myelin sheath or cross-reaction of antibodies against myelobasic protein may lead to impairment of myelin function (15).

In HCV-infected patients without cryoglobulinemia, the most common neurological manifestations are mononeuropathy and multineuropathy $(4,11)$. HCV RNA has been isolated in homogenates of nerve biopsy specimens in these patients by in situ PCR 
$(11,17)$. These findings suggest a direct role of $\mathrm{HCV}$ in the pathogenesis of peripheral neuropathy. Possible mechanisms include a direct nerve cytopathic effect by HCV or an immune-mediated mechanism, such as immune complex-induced changes of the epineurial vessel (11). Another possible mechanism is a humoral immune response combined with an imbalanced antigen/antibody ratio, resulting in excess of immune complexes, which, once deposited on the vessel walls, induce an inflammatory response by activation of the complement system. These inflammatory processes lead to lymphocytic and histiocytic infiltrations into small vessels and ischemic or inflammatory organ lesions (15).

There are no definitive guidelines for the treatment of $\mathrm{HCV}$-associated neurological manifestations. Some therapeutic options are immunosuppressive agents, such as corticosteroids and/or immunomodulating agents, e.g., IFN and ribavirin (18). Other studies have also shown clinical improvement of acute inflammatory episodes following treatment with corticosteroids and cyclophosphamide $(4,15,19)$.

IFN is a powerful modulator of the humoral and cellular immune responses; however, its mechanism of action is poorly understood. Reduction of viral replication rate (even without complete or permanent elimination), inhibition of lymphocyte proliferation and immunoglobulin synthesis, and improvement of immune complex clearance by enhancing macrophage activity may account for its therapeutic properties (18). Previous studies have shown that treatment with IFN as monotherapy resulted in improvement of vasculitis-associated symptoms and serum cryoglobulin levels, but symptoms rebounded after treatment discontinuation (20). In the present report, the patient showed complete and sustained remission of both HCV RNA and neurological symptoms after 48 weeks of treatment with IFN- $\alpha$ and ribavirin. We observed that neurological symptoms started to improve after 28 weeks of treatment, when the patient could have had negative or very low levels of HCV RNA. Unfortunately, HCV RNA determination was not performed at that time, but only at the end of treatment and 6 months later. Further studies are needed to document HCV RNA clearance and neurological symptom improvement.

The management of HCV-associated peripheral neuropathy should be based on its severity and on response to treatment. Corticosteroids and/or IFN plus ribavirin are the first options in patients with mild to moderate neuropathy (19). Since chronic corticosteroid administration is associated with increased serum cryoglobulin and HCV RNA levels, this drug should be administered in low doses and reduced as soon as possible. In addition, the long-term consequences of corticosteroid use for liver function of HCVinfected patients remain unknown and this class of drugs should be used with caution. For patients who do not respond to this initial approach, intravenous immunoglobulins can be considered. Plasmapheresis appears to be a reasonable option in severe or refractory cases. Even patients without active hepatic disease (normal alanine aminotransferase and mild hepatic lesion by liver biopsy) show improvement of cryoglobulinemia-associated neurological symptoms when treated with IFN plus ribavirin (20).

$\mathrm{HCV}$ infection is widespread throughout the world and can be associated with extrahepatic manifestations, such as the peripheral neuropathy case illustrated in this report. Patients with HCV infection should be investigated for neurological manifestations and HCV testing should be performed in patients with unexplained peripheral neuropathy. Finally, antiviral treatment with IFN plus ribavirin, corticosteroids, and/or neuromodulators may improve the neurological symptoms and should be considered in such patients. 


\section{Acknowledgments}

The authors would like to thank Silvia Lazarini, RN and Glaucia Quaresma for nursing care and logistic coordination, respectively, at the Grupo de Estudo das Hepatites Virais outpatient clinic, Hospital das Clínicas,
Universidade Estadual de Campinas, and Brady D. Beecham, Master's student in Public Health, Sree Chitra Tirunal Institute of Medical Sciences and Technology, Trivandrum, India, for reviewing the manuscript.

\section{References}

1. Lauer GM \& Walker BD (2001). Hepatitis C virus infection. New England Journal of Medicine, 345: 41-52.

2. Gordon SC (1996). Extrahepatic manifestations of hepatitis C. Digestive Diseases, 14: 157-168.

3. Zaltron S, Puoti M, Liberini P et al. (1998). High prevalence of peripheral neuropathy in hepatitis $C$ virus infected patients with symptomatic and asymptomatic cryoglobulinaemia. Italian Journal of Gastroenterology and Hepatology, 30: 391-395.

4. Lidove O, Cacoub P, Maisonobe T et al. (2001). Hepatitis C virus infection with peripheral neuropathy is not always associated with cryoglobulinaemia. Annals of Rheumatic Diseases, 60: 290-292.

5. Gumber SC \& Chopra S (1995). Hepatitis C: a multifaceted disease. Review of extrahepatic manifestations. Annals of Internal Medicine, 123: 615-620.

6. Cacoub P, Renou C, Rosenthal E et al. (2000). Extrahepatic manifestations associated with hepatitis $C$ virus infection. A prospective multicenter study of 321 patients. Medicine, 79: 47-56.

7. Lunel F \& Cacoub P (1999). Treatment of autoimmune and extrahepatic manifestations of hepatitis $C$ virus infection. Journal of Hepatology, 31 (Suppl 1): 210-216.

8. Lunel F, Musset L, Cacoub P et al. (1994). Cryoglobulinemia in chronic liver diseases: role of hepatitis $\mathrm{C}$ virus and liver damage. Gastroenterology, 106: 1291-1300.

9. Agnello V, Chung RT \& Kaplan LM (1992). A role for hepatitis C virus infection in type II cryoglobulinemia. New England Journal of Medicine, 327: 1490-1495.

10. Hadziyannis SJ (1998). Skin diseases associated with hepatitis C virus infection. Journal of the European Academy of Dermatology and Venereology, 10: 12-21.

11. Nemni R, Sanvito L, Quattrini A et al. (2003). Peripheral neuropathy in hepatitis C virus infection with and without cryoglobulinaemia. Journal of Neurology, Neurosurgery, and Psychiatry, 74: 1267-1271.

12. Migliaresi S, Di lorio G, Ammendola A et al. (2001). Peripheral nervous system involvement in HCV-related mixed cryoglobulinemia. Reumatismo, 53: 26-32.

13. Gemignani F, Melli G, Inglese $C$ et al. (2002). Cryoglobulinemia is a frequent cause of peripheral neuropathy in undiagnosed referral patients. Journal of the Peripheral Nervous System, 7: 59-64.

14. Costa J, Resende C \& de Carvalho M (2003). Motor-axonal polyneuropathy associated with hepatitis $\mathrm{C}$ virus. European Journal of Neurology, 10: 183-185.

15. Heckmann JG, Kayser C, Heuss D et al. (1999). Neurological manifestations of chronic hepatitis C. Journal of Neurology, 246: 486491.

16. Tembl JI, Ferrer JM, Sevilla MT et al. (1999). Neurologic complications associated with hepatitis $C$ virus infection. Neurology, 53: 861864.

17. Authier FJ, Bassez G, Payan C et al. (2003). Detection of genomic viral RNA in nerve and muscle of patients with HCV neuropathy. Neurology, 60: 808-812.

18. Khella SL, Frost S, Hermann GA et al. (1995). Hepatitis C infection, cryoglobulinemia, and vasculitic neuropathy. Treatment with interferon alpha: case report and literature review. Neurology, 45: 407411.

19. Ramos-Casals M, Trejo O, Garcia-Carrasco M et al. (2003). Therapeutic management of extrahepatic manifestations in patients with chronic hepatitis C virus infection. Rheumatology, 42: 818-828.

20. Misiani R, Bellavita P, Fenili D et al. (1994). Interferon alpha-2a therapy in cryoglobulinemia associated with hepatitis $C$ virus. New England Journal of Medicine, 330: 751-756. 